\title{
SHELLS AND THE POTENTIAL WELLS OF ELLIPTICAL GALAXIES
}

\author{
P.J.Quinn ${ }^{1}$ and Lars Hernquist ${ }^{2}$ \\ 1. Space Telescope Science Institute, Baltimore, MD 21218. \\ 2. Dept. of Astronomy, Univ. California, Berkeley, CA 94720.
}

\begin{abstract}
A survey of the possible variety of sharp-edged, caustic features that may arise in the collision of galaxies with very different masses and sizes (Hernquist and Quinn 1986a) has shown that in general shells are morphologically very complex. It is therefore not easy to determine the history of the collision that produced the shells nor the properties of the galaxies involved. However, a small number of shell galaxies (notably NGC 3923) have a sufficiently simple and orderly shell distribution that we believe the shells were formed by a chance very symmetric and simple encounter. In such cases we are presented with a unique opportunity to investigate the potential well of an elliptical galaxy over a large range in radius $\left(\simeq 0.5 r_{e}-20 r_{e}\right)$. An analysis of the NGC 3923 shell system (Hernquist and Quinn 1986b) has shown that a large amount of dark matter is present $\left(M_{\text {dark }} \simeq 40 M_{\text {luminous }}, r<17 r_{e}\right)$.
\end{abstract}

\section{SHELL HISTORY.}

Over the past six years, our knowledge of shell systems has grown from a handfull of examples to a point where shells are now considered a common feature of field ellipticals. The word "shell" was first used by Arp (1966) to classify some of the features he saw in ARP 230. The Arp atlas has proved to be an invaluable source of information on the variety and abundance of interacting systems and has provided much of the fuel for the growing idea that galaxies may have been severly modified by mergers during their lifetimes (Toomre 1977). Further examples of shells were slow in coming after the Arp Atlas; Dave Malin's work on M89 being a notable addition (Malin 1979). The full implications of shells and their connection to interactions involving disk galaxies was first pointed out by Francois Schweizer in his work on Fornax A (Schweizer 1980). Schweizer discovered prominant sharpedged features (which he called "ripples") in the outer optical envelope of Fornax A and concluded correctly that such sharp stellar features could not be formed from the "hot" stellar population that made up the main body of Fornax A. Rather, this material must have been introduced from another "colder" galaxy. At this point the connection between peculiar galaxies, mergers and shells had been made. However the real surprise was the keystone work of Malin and Carter (1980) who demonstrated the existence of shells in otherwise completely normal looking ellipticals. The discovery of a beautiful set of very symmetric shells around NGC 3923 was achieved by applying photographic enhancement techniques to U.K. Schmidt IIIaJ survey plates and A.A.T. prime focus plates. These techniques were largely 
developed for astronomical applications by Malin (Malin 1981,1982). The existence of low light level shells around otherwise normal ellipticals means that shells are possibly a very common and relatively long lived component of many ellipticals and implies mergers are a fundamental part of the history of elliptical galaxies.

Subsequent surveys for shells (Malin and Carter 1983, Schweizer and Ford 1985) have turned up over 200 examples of shell systems. Very few of these galaxies have been examined photometrically and spectroscopically but in the few cases that have, the general conclusion is that shells are indeed stellar; they have colors that are close to that of the parent and the parents themselves are kinematically and chemically like shell-less ellipticals (Carter, Allen and Malin 1982; Quinn 1982; Fort et. al. 1985; Pence 1986; ,Wilkinson, Sparks, Malin and Carter 1986). The proposition that some well studied peculiar ellipticals (like Cen. A) were the result of a merger gained support by finding telltale shells in their outer luminous envelopes (Malin, Quinn and Graham 1983).

On the theoretical side, Fabian, Nulsen and Stewart (1980) were the first to propose an origin for the shells reported by Malin and Carter (1980). Their theory called for shells being formed at shocks in an outflowing wind (a similar theory has also been proposed by Williams and Christiansen (1985)). At present, wind related theories seem to be less consistent with the observations than those involving mergers (see Athanassoula and Bosma 1986 for a review). Following Schweizer's suggestion of a merger related origin for shells, Quinn (1982, working with Agris Kalnajs) was able to produce sharp edged features in collisions involving disks and massive ellipticals that were qualitatively very similar to those found by Malin and Carter. Subsequent work by Toomre (1983), Quinn (1984), Dupraz and Combes 1986, and Hernquist and Quinn (1986 a,b) has shown that mergers and accretions can very naturally account for the diversity of the observed shell galaxies. Shell making is a very robust and inevitable consequence of the destruction and cannibalism of a small and/or cold companion galaxy by a massive parent. Further studies are reported elsewhere in this volume (James and Wilkinson 1986, Piran and Villumsen 1986).

\section{LAYING TO REST SOME MISCONCEPTIONS ABOUT SHELLS.}

Like all good prototypes in astronomy, NGC 3923 and the first sample of shell systems presented by Malin and Carter (1980) have proven to be atypical of the majority of the shell systems now known. Hence, by attempting to model the features of NGC 3923, the initial theoretical work of Quinn $(1982,1984)$ gave a rather narrow view of the way shell systems might be formed. In particular, Quinn found that a nearly radial orbit for the companion was prefered if the shells were to be aligned like those of NGC 3923. Nonradial companion orbits gave rise to complex and interpenetrating structures that were unlike the shells in NGC 3923. Also Quinn neglected the possibility that a small spheroidal companion could be as "cold" as the disk-like companions proposed by Schweizer to be responsible for the shells.

Hernquist and Quinn (1986a) have conducted a fairly complete survey of the possible parameter space taking into account progressive disruption of the companion, disk and spheroidal companions, and non-spherically symmetric parents. Their conclusion was that shell-like features can be formed during the accretion $\left(\Delta E_{\text {orbit }}=0\right)$ or merger $\left(\Delta E_{\text {orbit }}<0\right)$ events involving a fixed primary galaxy 
(either spiral or elliptical) and a small and/or cold companion. Companions have to have a small phase space volume relative to the primary in order to make many sharp shells. Hence the companion can be either spatially smaller than the primary, or have a smaller velocity width than the primary (colder) or both. Shell-making is therefore a very robust dynamical process and should be the rule rather than the exception.

The diversity of structures found in the Hernquist and Quinn models complements the observed diversity of morphologies in the Malin-Carter Catalogue (Malin and Carter 1983). The typical shell galaxy may have a small number of non-concentric, non-aligned, interpenetrating shells as well as radial filaments. In a case like NGC 3923 which has aligned, very symmetric, simple shells, the companion must have had a simple morphology and the orbit must have been very nearly radial (see Figure 1). Non-radial encounters involving either disks or elliptical companions and radial encounters involving disks, produce shells that are very different from those observed in NGC 3923 (see Figure 2). Hence NGC 3923-like systems should be rare amongst the shell galaxies (which they are) and they may afford us a particular simple case in which to test and use the phase-wrapping theory of shell formation (Quinn 1984).

\section{MAKING SOME SENSE OUT OF THE DIVERSITY OF SHELL GALAXIES.}

Shells were defined by Malin and Carter as "edge-brightened structures in the optical envelope or (more often) beyond the discernible limits of the elliptical galaxy". Most of the structures formed in the simulations of Hernquist and Quinn would certainly be classified as "shells" by the above definition, yet geometrically they may be totally unlike a shell. Clearly the label "shell" should not be taken in its literal sense but rather as a catch-all for a large class of sharp-edged stellar features which are not necessarily of constant curvature.

One clear systematic trend evident in the Hernquist and Quinn simulations is that the most complex looking structures are always associated with disk-like companions. The fact that the companion is sheet-like, permits folding of the companion in configuration space as well as phase space. The fold catastrophies that result when the distorted disk-like companion is projected onto the plane of the sky appear as extended, bright, sharp-edged features of the types classified by Arnold (1984). This would then imply that in those cases where we see a complex shell morphology (e.g. Cen. A, NGC 2685) we should also expect to see other signs of a disk-like intruder such as gas and dust. In all the examples known to the authors, this is observed to be the case.

The theory of catastrophic processes has direct implications for the photometry of shells. The fall-off of density behind a simple fold caustic is proportional to $x^{-\frac{1}{2}}$ ( where $x$ is the distance from the caustic) regardless of the form of the potential well in which the system evolves. Hence for a "shell" produced by the spatial folding of a disk we would expect the luminosity to decline like $x^{-\frac{1}{2}}$ giving the shell a bright edge and rapid fall off towards the galaxy. If, however, the shell was produced by the phase folding of a spheroidal companion, then the $x^{-\frac{1}{2}}$ behaviour occurs when the complete phase space is projected on the spatial (radial) axis. When this distribution is projected on the sky, the resultant luminosity profile is nearly flat, giving the shell a plateau-like appearance. Both types of photometric behavior have been observed (Pence 1986, Fort et. al. 1985). We would expect the 

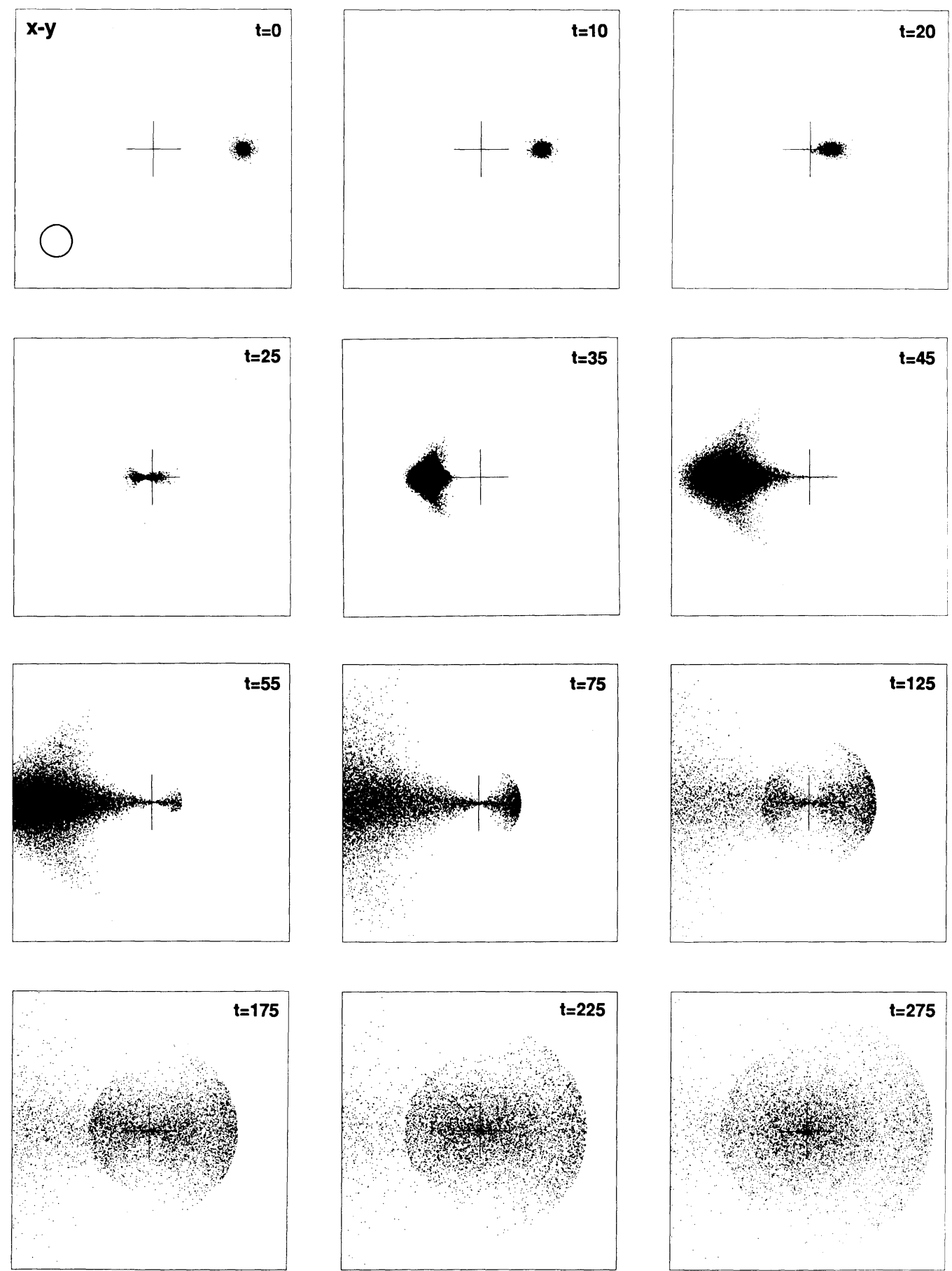

Figure 1. A radial encounter between a spherical Plummer primary and a spherical companion. The companion mass was 0.01 and its half-mass radius was 0.2 (both 1 for primary). The circle in the first frame indicates a spherical primary was used and the cross is at the center-of-mass. 


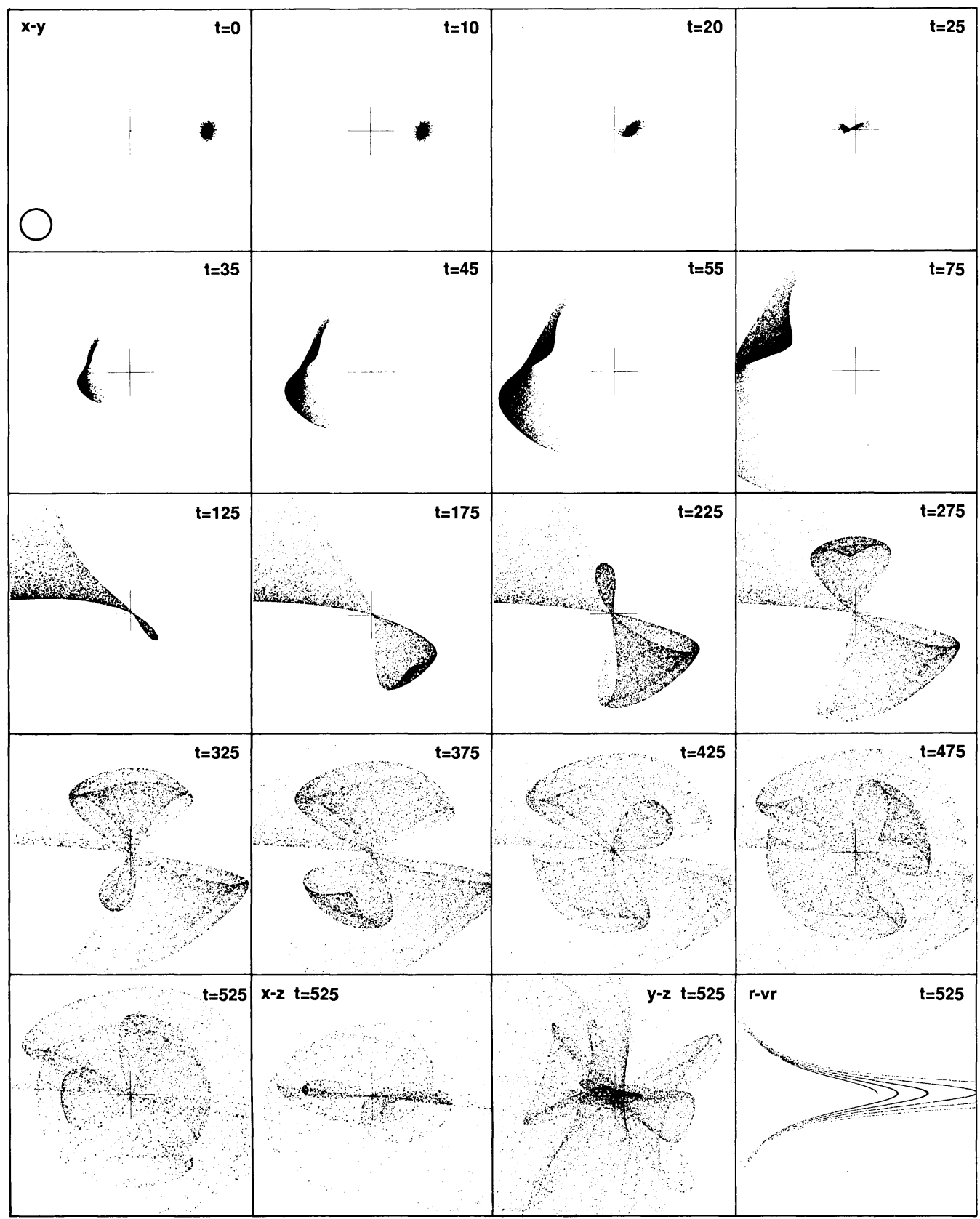

Figure 2. A radial encounter between a spherical Plummer primary and an exponential disk with an inclination of $45^{\circ}$ to the $x-y$ plane. The disk has a mass of 0.01 . The final panel shows the phase space distribution of particles at $t=525$. The box dimensions are $40 \times 40$ as in Figure 1 . The time units are $4.3 \times 10^{6}$ years for a half-mass radius of $2 \mathrm{kpc}$. and a total mass of $10^{11} M_{\odot}$. 
complex shell systems to show the strongly edge-brightened behaviour as it is most likely that these encounters involve disk-like companions.

Finally, if very little orbital energy is lost by the companion before it is destroyed (an accretion) then the spread in energies of the shell stars will reflect that initially present in the companion. If the orbital energies are close to zero then it is unlikely that shell stars would have turning-radii closer than a few half-light radii of the elliptical parent provided the companion is much less massive than the parent. Several shell galaxies have shells at radii as small as $0.5 r_{e}$ which means that either the companion was quite massive (i.e. had a large spread in internal energies) or a significant fraction of the companions orbital energy was lost by dynamical friction on the parent before the companion was destroyed (a merger). The luminosity of the shell material is difficult to estimate but a reasonable guess seems to be less than $5 \%$ of the parent luminosity (Pence 1986). It would therefore seem likely that the companions involved were not very massive compared to the parent. This would then imply that dynamical friction had been at work in those systems with inner shells. Given a constraint on the luminous mass of the companion from the shell luminosity, the observed range in shell radii and some constraints on the orbit of the companion, limits can be placed on the work done by friction and hence the amount of dark material present. Clearly if one knew the number of shell systems with inner shells, the shell luminosities and morphologies, then useful constraints could be placed on dark matter in ellipticals. Most likely self-consistent simulations are called for to get the orbital evolution and disruption process right (James and Wilkinson, this volume).

\section{NGC 3923 AS A SIMPLE SHELL GALAXY.}

If the shells in NGC 3923 were produced by an encounter with a elliptical companion on a nearly radial orbit then the resultant shells are formed by the phase wrapping mechanism. In this process, the companion is drawn out and wrapped up in phase space by the difference in radial periods between the most bound and least bound stars. The number of wraps that occur by a time $t$ after the companion is destroyed is given approximately by :

$$
N \sim t\left(\frac{1}{P_{\min }}-\frac{1}{P_{\max }}\right)
$$

If we measure the time passed in units of the maximum period $\tau=\frac{t}{P_{\max }}$ then

$$
N \sim \tau\left(\frac{P_{\max }}{P_{\min }}-1\right)
$$

where $P$ is the radial period. If we model NGC 3923 as a $r^{\frac{1}{4}}$ law mass profile then given an outer shell radius $d_{\max } \sim 16 r_{e}$ and an inner shell radius $d_{\min } \sim 0.6 r_{e}$, the above equation would predict $N_{r} \sim 106$ if $\tau$ has its minimum value. However the observed number of shells between these two radii is $\sim 20$. We could solve this discrepancy by either decreasing $P_{\max }$ or increasing $P_{\min }$. Since NGC 3923 does follow an $r^{\frac{1}{4}}$ law luminosity profile we are confident about $\boldsymbol{P}_{\text {min }}$. So we are forced to decrease $P_{\max }$ by a factor of order 6 or equivalently we must increase the 
mass at large radius by a factor $\sim 6^{2}$ since $P(r) \sim M(r)^{-\frac{1}{2}}$. Making the system older ( $\tau$ larger) only increases the discrepancy. Hence the relatively small number of shells in the outer envelope of NGC 3923 given the spread in radius, implies a large amount of dark matter $\left(M_{\text {dark }} \sim 40 M_{\text {lum. }}\right.$.) out to a radius $\sim 100 h^{-1} k p c$.

A more detailed analysis of the shell distribution has been conducted by Hernquist and Quinn(1986b). They conclude that if the halo has a density distribution like that of a non-singular, isothermal sphere then the halo mass out to $100 \mathrm{~h}^{-1} \mathrm{kpc}$. is $40 M_{\text {lum }}$. and the core radius $\gamma \sim 3 r_{e}$. There are uncertainities and difficulties with the analysis of NGC 3923. Firstly to correctly establish the relative amount of dark matter present, the age of the shell system $(\tau)$ must be known. Allowing $\tau$ to be greater than its minimum value increases the amount of dark matter required. Fortunately $\tau$ for NGC 3923 is constrained from above by requiring the luminous material to be self-gravitating (see Hernquist and Quinn 1986 for details). Secondly, there are missing shells in NGC 3923. Gaps are expected since the shell brightness distribution depends on the spatial and velocity distributions in the companion and the shape of the parent potential. Irregularities in position or velocity in the companion or in the potential well of the parent will be reflected as missing or faint shells. Thirdly, what influence has dynamical friction had on the shell distribution? To answer this completely will require detailed, self-consistent simulations. It is hoped that by avoiding the very innermost shells, we are dealing with a set of shells that were all produced from the same disruption event rather than from multiple passages of a single companion as its orbit decays or multiple companions. Bearing these difficulties in mind, simple shell galaxies like NGC 3923 present us with a unique opportunity to probe the potentials of ellipticals.

\section{THE HALOS OF ELLIPTICALS AND SPIRALS}

Given that the dark halos of spirals and ellipticals behave like isothermal spheres at large radii, we can characterize the halos by either their asymptopic rotational velocity or the slope of their mass profile :

$$
\frac{M}{r}=\frac{V_{A}}{G}=\text { constant }=\Xi
$$

The constant $\Xi$ is directly proportional to the depth of the halo potential and the mean halo density within some given radius. For NGC 3923 we know that approximately 40 times as much dark matter as bright is contained within the outer shell radius $\left(r \sim 100 h^{-1} k p c\right.$.) giving $\Xi \sim 6 \times 10^{7} M_{\odot} p c^{-1}$. A similar number can be obtained from the $\mathrm{X}$-ray sample of early type galaxies by Forman, Jones and Tucker (1985). Assuming that the maximum radius at which $\mathrm{X}$-rays are detected is well outside the core radius of the dark matter then $\langle\Xi\rangle \sim 5 \times 10^{7} M_{\odot} p c^{-1}$ in good agreement with the shell data. For spirals, we can estimate $\Xi$ from the Bahcall and Casertano sample (Bahcall and Casertano 1985) or the Tully-Fisher relationship again assuming that the velocities truly represent the asymptopically flat values. We obtain : $\langle\Xi\rangle \sim 0.7 \times 10^{7} M_{\odot} p c^{-1}$ (see Hernquist and Quinn 1986c, Figure 3, this volume). This would then imply that ellipticals may have typical halo densities which are perhaps an order of magnitude larger than that of spirals. Some caution is necessary however, since the overlap in luminosity between the X-ray sample and the spirals is very small. Hence it is difficult to compare spirals and ellipticals at the same luminous mass until X-ray data can be taken on less luminous ellipticals. 
However, since the luminosity density of ellipticals is approximately ten times that of spirals, an indication of a similar ratio for their dark matter would favour theories in which the halos of galaxies strongly influence the type of galaxy that forms within them.

\section{REFERENCES}

Arnold, V.I., 1984, Catastrophe Theory, Springer Verlag.

Arp, H., 1966, Atlas of Peculiar Galaxies, Astrophys. J. Suppl., 123, 1.

Athanassoula, E., \& Bosma, A., 1985, Ann. Rev. Astr. Astrophys., 23, 147.

Bahcall, J.N., \& Casertano, S., 1985, Astrophys. J. Letters, 293, L7.

Carter, D., Allen, D.A., \& Malin, D.F., 1982, Nature, 295, 126.

Dupraz, C., \& Combes, F., 1986, Astr. Astrophys., 166, 53.

Fabian, A.C., Nulsen, P.J., \& Stewart, G.C., 1980, Nature, 287, 613.

Forman, W., Jones, C., \& Tucker, W., 1985, Astrophys. J., 293, 102.

Fort, B.P., Prieur, J.L., Carter, D., Meatheringham, S.J., \& Vigroux, L., 1986, Astrophys. J., 306, 110.

Hernquist, L., \& Quinn, P.J., 1986a, Astrophys. J., submitted.

Hernquist, L., \& Quinn, P.J., 1986b, Astrophys. J., in press.

Hernquist, L., \& Quinn, P.J., 1986c, this volume, p. 467.

James, R.A., \& Wilkinson, A., 1986, this volume, p. 471.

Malin, D.F., 1979, Nature, 277, 279.

Malin, D.F., 1981, J. Photo. Sci., 29, 199.

Malin, D.F., 1982, J. Photo. Sci., 30, 87.

Malin, D.F., \& Carter, D., 1980, Nature, 285, 643.

Malin, D.F., \& Carter, D., 1983, Astrophys. J., 274, 534.

Malin, D.F., Quinn, P.J., \& Graham, J.A., 1983, Astrophys. J. Letters, 272, L5.

Pence, W., 1986, STScI preprint.

Piran, T., \& Villumsen, J.V., 1986, this volume, p. 473.

Quinn, P.J., 1982, Ph.D. Thesis, Australian National University.

Quinn, P.J., 1984, Astrophys. J., 279, 596.

Schweizer, F., 1980, Astrophys. J., 252, 303.

Schweizer, F., \& Ford, W.K.Jr., 1985, in New Aspects of Galaxy Photometry, Lecture Notes in Physics No. 232, ed. J.-L. Nieto (Springer Verlag), p. 145.

Toomre, A., 1977, In The Evolution of Galaxies and Stellar Populations, eds B. Tinsley \& R. Larson, (Yale Univ. Obs.), p. 401.

Toomre, A., 1983, in IAU Symposium No. 100, The Internal Kinematics and Dynamics of Galaxies, ed. E.O. Athanassoula, (Reidel, Dordrecht), p. 319.

Wilkinson, A., Sparks, W.B., Malin, D.F., \& Carter, D., 1986, this volume, p. 465.

Williams, R.E., \& Christiansen, W.A., 1985, Astrophys. J., 291, 80. 


\section{DISCUSSION}

Ostriker: i) Do I recall correctly that at last year's dark matter symposium you said that in order to fit the observations you required that there be no massive halo, that the mass given by $\mathrm{X}$-ray observations be wrong, too high? Now I understand you to say that the $\mathrm{X}$-ray observations confirm the model. ii) If the shells add up to $10 \%$ of the light, then from the Faber-Jackson relation, a velocity dispersion of the captured object would be $50 \%$ of the cannibal. Is this cold enough to give you the observed sharp features?

Quinn: As regards the paper I gave with Wojcek Zurek and John Salman at IAU 117 on Dark Matter, we concluded that it was not possible to fit the shell distribution in NGC 3923 without dark matter. What was in doubt at that time was the distribution of the dark matter at large radii. Lars Hernquist and I have now clarified this in a recent preprint. Whether or not a given companion can produce a given number of shells before smearing sets in, depends not only on the velocity dispersion in the companion relative to the primary, but also on its physical size. In other words, the phase-space volume of the companion has to be small compared to the primary. Hence a small hot companion can produce shells. One should also note that shells sharpen as time goes by due to the system being stretched in phase. I believe that a $50 \%$ velocity dispersion ratio is not a problem, provided the sizes have a ratio of greater than about ten to one, which does not seem to be unreasonable. It is my impression from the work of Bill Pence on NGC 3923, that the shells represent more like $2 \%$ of the total luminosity of this galaxy.

Fabian: I think that perhaps Alar Toomre and you misunderstand my comment yesterday on producing shells in cooling flows. The stars produced there are very cold $(v \sim 10 \mathrm{~km} / \mathrm{s})$ and much colder than any galaxy.

Quinn: Yes, such cold stars could produce shells, but if they formed with the body of the main galaxy they could not produce shells at large radii.

Binney: Did I understand that in your shell-counting argument you assumed that all shells started at the same instant? I should have thought that the outer shells got a head start by one free-fall time, since they would form as the victim started to fall in, while the inner shells would form only after the victim had been dragged, kicking and screaming, to the center.

Quinn: The shell counting argument is not very sensitive to the distribution of initial orbital phases. If the companion disrupts on the first pass then differences in phase between lightly and loosely bound stars are quickly forgotten as the most bound stars complete a large number of periods in one crossing time of the least bound stars. Even if I forbid the most tightly bound stars from making shells at all for a time comparable to the period of the least bound stars, the corrections to the shell numbers are $\sim 2 / 3$ which still leaves us with a factor of 5 discrepancy between the observed shell distribution and that predicted by the Kepler formula.

Bland: Would you not expect the shells to be interleaved in radius with respect to the deep potential and is this observed for the most part in the Malin \& Carter catalogue? 
Quinn: NGC 3923 does show interleaving as do a few others I know of (NGC 3051, Arp 230 etc.). As far as the whole catalogue goes we will have to wait for Althea Wilkinson to reduce her beautiful new CCD data.

Nieto: Some of your simulations show very complex inner structures that are very reminiscent of the structure of dusty or box-shaped ellipticals, once the low luminosity gradient has been taken off. Do you think that this could mean a relationship between dust and ripples? In other words, do you think that the dust and/or the box-shapes in ellipticals may be a remnant of a merger implying a spiral galaxy?

Quinn: Certainly I would expect complex (crossing, nonconcentric) shells to be associated with the distruction of a disk-like companion. Hence in such cases dust and gas should be present. In the case where the primary is axisymmetric, the orbital plane of the companion precesses about the symmetry axis and can produce $\mathrm{X}$-like structures as have been seen in spiral bulges. "Boxy-ness" in ellipticals may be due to contributions from debris in an $\mathrm{X}$-shaped configuration.

Freeman: You showed a run with a triaxial potential that gave nice well aligned shells like those of NGC 3923. If there were a high incidence of triaxial potentials, might one expect more shell systems like NGC 3923's?

Quinn: The problem is that even when the parent potential is strongly triaxial, the form of the shells depends also on the type of the companion and its orbital parameters. For example, an inclined disk-like companion or even a radial orbit does not produce a clean set of aligned shells when thrown into a triaxial parent. Similarly a spheroidal companion falling down the short axis produces shells which are completely misaligned. It is therefore dangerous to draw conclusions about parent shapes from the shell alignment alone.

Whitmore: If your need for. a heavy halo in NGC 3923 is solely reliant on being able to identify all $\approx 120$ shells you predict in your model with no halo, the observational difficulty of actually identifying that many very faint features will probably be the biggest problem. However, if the qualitative look of the shells and their distribution in radius is also better fit by the heavy halo model, this would be more convincing.

Quinn: If there were even twice as many shells present as observed, I'm sure they could be easily seen on the plates. I agree that the shell distribution is the telling factor for the dark matter.

Bertin: Is the self-gravity of the infalling disks included in your simulations?

Quinn: Not in the simulations with Hernquist. In my Ph.D. thesis I did some self-gravitating simulations and showed that it was not important for shell making. Shell making (phase wrapping) is a test particle process, self-gravity just regulates the rate of release of free particles into the primary potential.

Combes: Simulations show that around a prolate galaxy, shells are usually seen aligned along the apparent major axis. The prototype case is NGC 3923. But when a strong figure rotation is introduced (tumbling period short in front of companion 
stars oscillation periods), the alignment disappears. The prolate potential is timeaveraged in an oblate potential for the shells and one can see randomly spread shells in the plane perpendicular to the rotation axis - this means that NGC 3923 is tumbling very slowly, if at all.

Quinn: I agree. The period of stars in the outermost shells is very long $\left(\geq 5 \times 10^{8}\right.$ yrs) for NGC 3923 . Hence even a very slow tumble rate could be ruled out $\left(t_{\text {tumble }} \geq\right.$ $5 \times 10^{9} \mathrm{yrs}$ ). Observations along the major axis of NGC 3923 I made in my thesis showed no apparent rotation at the $\pm 30 \mathrm{~km} / \mathrm{s}$ level.

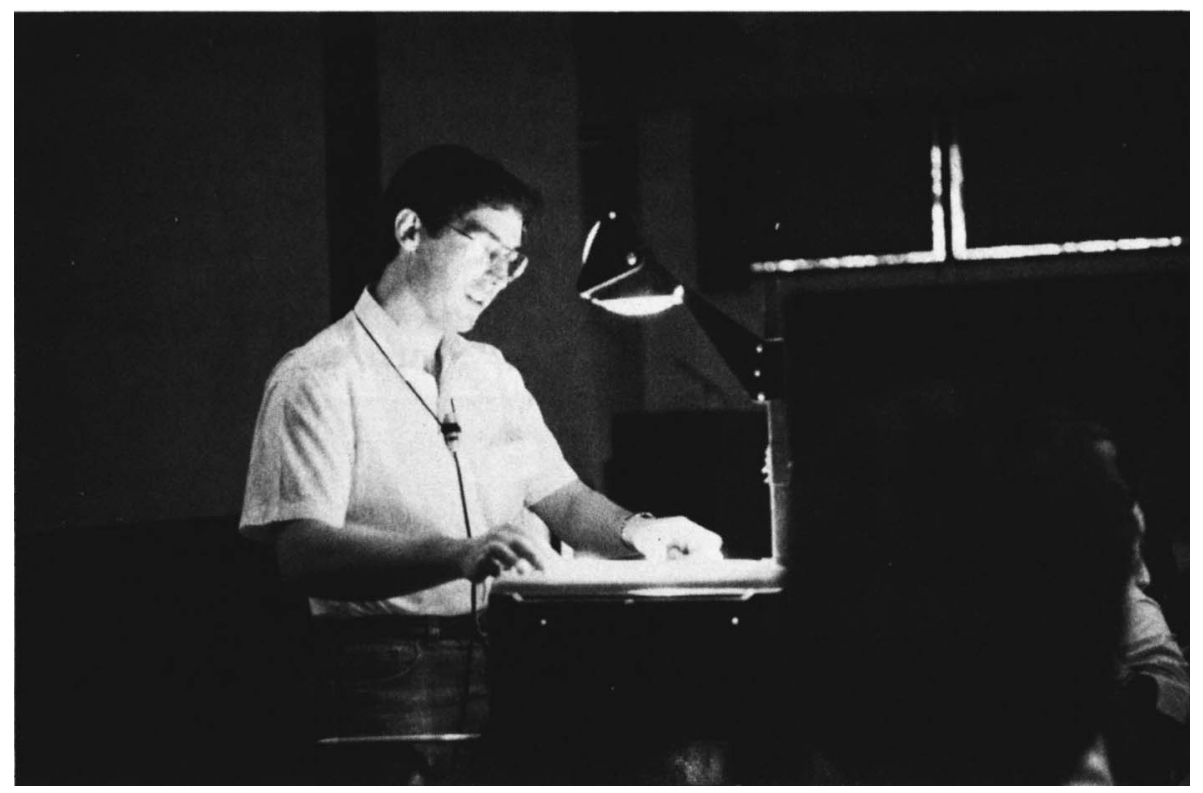

Peter Quinn. 


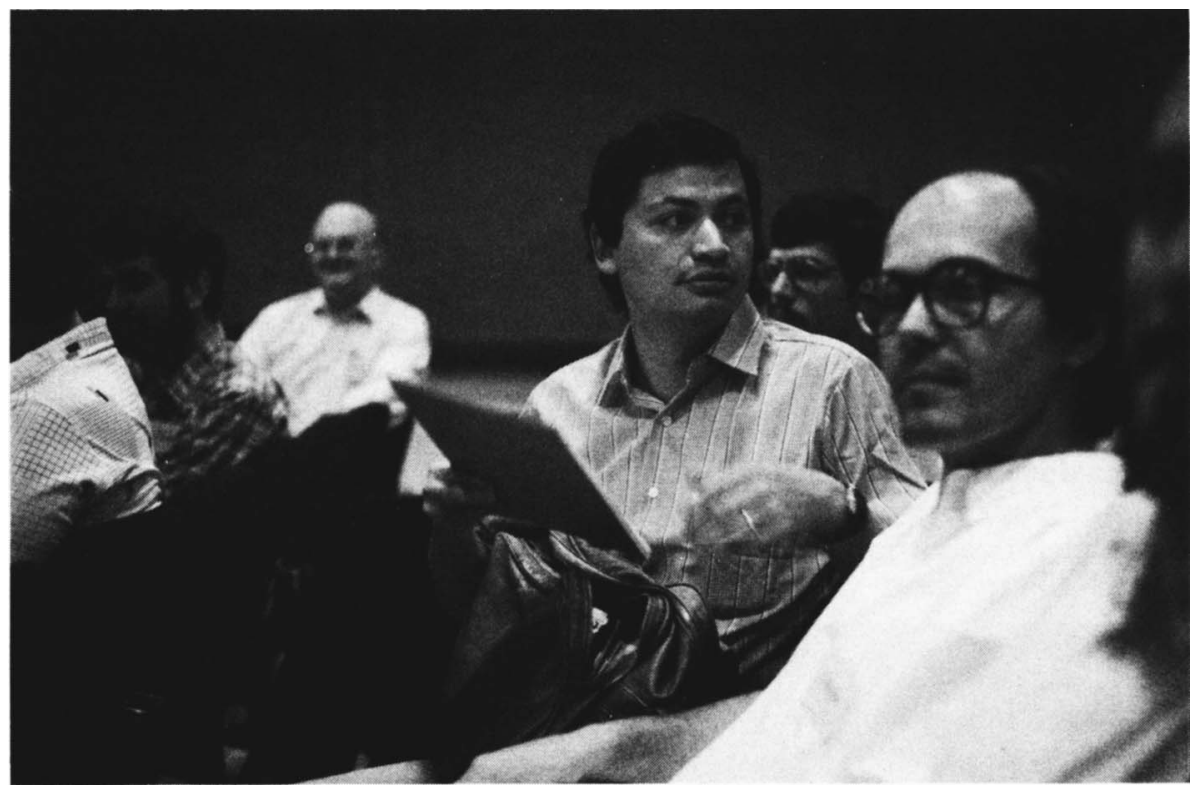

Aguilar, with Meylan on his right, and on his left Schechter and Toomre.

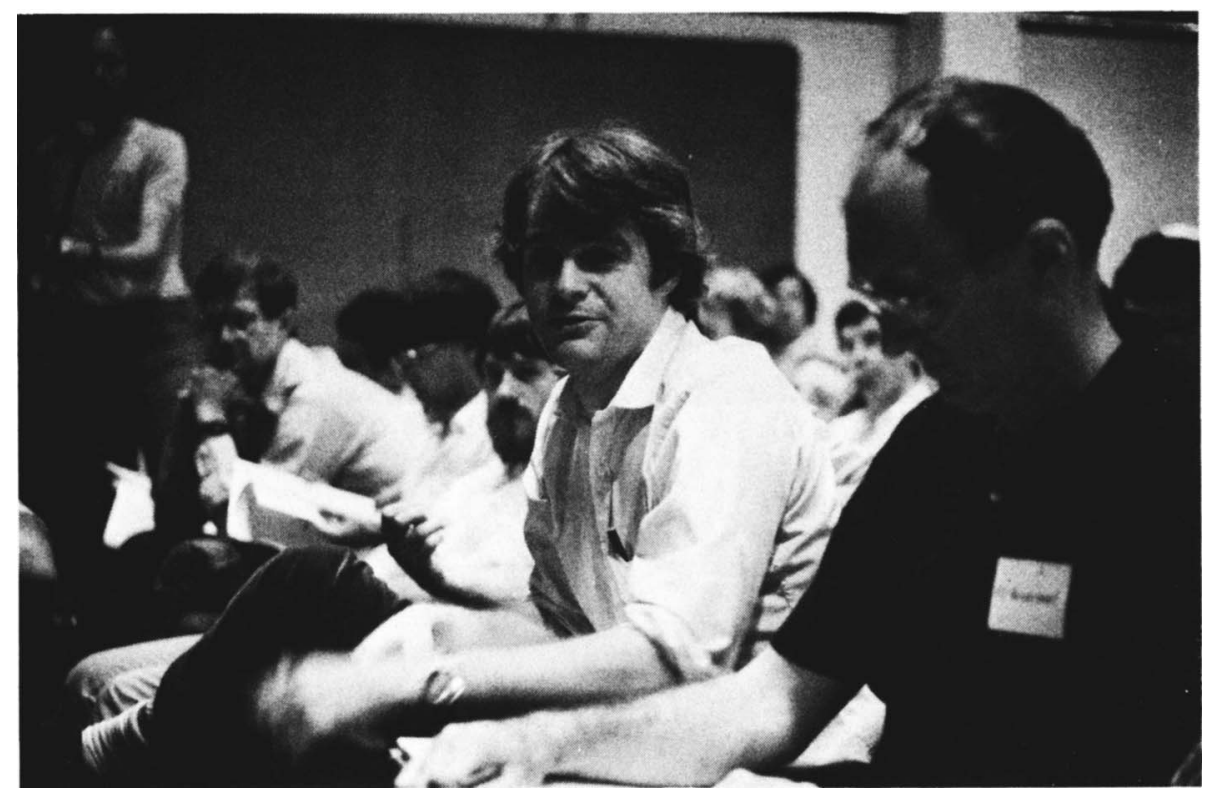

Lauer, with next to him Vandervoort. 